\title{
Retraction Note to: CXCR4 Signaling Induced Epithelial-Mesenchymal Transition by PI3K/AKT and ERK Pathways in Glioblastoma
}

\author{
Baoyu Lv ${ }^{1}$ Xiangshan Yang ${ }^{2} \cdot$ Shunzeng $\mathrm{Lv}^{2,3} \cdot$ Lei Wang ${ }^{4} \cdot$ Kaixi Fan $^{5}$.

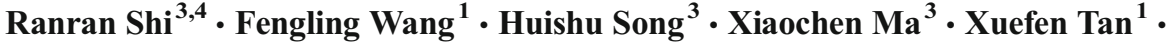 \\ Kun $\mathrm{Xu}^{1} \cdot$ Jingjing Xie ${ }^{5}$ - Guangmei Wang ${ }^{5} \cdot$ Man Feng $^{2} \cdot$ Li Zhang $^{6}$
}

(C) Springer Science+Business Media New York 2017

\section{Retraction Note to: Mol Neurobiol (2015) 52:1263-1268 DOI 10.1007/s12035-014-8935-y}

This article has been retracted at the request of the Editor-inChief and the Publisher per the Committee on Publication Ethics guidelines. The article shows evidence of irregularities in authorship during the submission process, and there is strong reason to believe that the peer review process was compromised and the article shows similarities with the following articles which were submitted within a close timeframe:

The online version of the original article can be found at http:// dox.doi.org10.1007/s12035-014-8935-y

Li Zhang

sdzhanglisd@163.com

1 Department of Oncology, Shandong Cancer Hospital and Institute, Jinan, China

2 Department of Pathology, Affiliated Hospital of Shandong Academy of Medical Sciences, Jinan, Shandong, China

3 Shandong University School of Medicine, Jinan, Shandong, China

4 Department of Thoracic Surgery, Shandong Provincial Hospital Affiliated to Shandong University, Jinan, Shandong, China

5 Department of Oncology, Affiliated Hospital of Shandong Academy of Medical Sciences, Jinan, Shandong, China

6 Department of Gynecologic Oncology, Shandong Cancer Hospital and Institute, No, 440 Jiyan Road, Jinan, Shandong 250117, China
Hao Yu, Linlin Zhang, Peishu Liu, CXCR7 signaling induced epithelial-mesenchymal transition by AKT and ERK pathways in epithelial ovarian carcinomas, Tumor Biology March 2015, Volume 36, Issue 3, pp 1679-1683, DOI: 10.1007/s13277-014-2768-1; Received: 7 October 2014

Anyan Liao, Weijie Wang, Dawei Sun, Yuliang Jiang, Suqing Tian, Jinna Li, Xiangshan Yang, Ranran Shi, Bone morphogenetic protein 2 mediates epithelialmesenchymal transition via AKT and ERK signaling pathways in gastric cancer, Tumor Biology April 2015, Volume 36, Issue 4, pp 2773-2778, DOI: $10.1007 /$ s13277-014-2901-1; Received: 11 October 2014

The article "CXCR4 Signaling Induced EpithelialMesenchymal Transition by PI3K/AKT and ERK Pathways in Glioblastoma" was received: 15 September 2014.

As such, the validity of the content of this article cannot be verified. 